\title{
Demanda de formación en profesionales sanitarios
}

\author{
F. Bosch a,b, E. Serés ${ }^{a}$
}

Son muchas las publicaciones que recogen reflexiones sobre diversos problemas en torno a la educación médica. Sin embargo, a menudo se dan por sabidas cuáles son realmente las necesidades de formación del profesional sanitario $[1,2]$. Un artículo publicado en una revista clínica de amplia difusión en España recoge la opinión de profesionales sanitarios respecto a las necesidades de formación continuada en temas de investigación [3]. A través de los resultados que presentan los autores, destaca la elevada motivación de este colectivo por formarse en determinadas áreas específicas, con diferencias en las preferencias según su experiencia individual. Según este estudio [3], los cursos más demandados por el profesional sanitario son sobre inglés científico, sobre redacción de artículos y sobre presentación en público. Al hilo de estas reflexiones, creemos que podría resultar de interés presentar la información que se recopiló mediante una encuesta realizada entre los asistentes a unos cursos de formación organizados desde la Fundación Dr. Antonio Esteve (FDAE).

En colaboración con otras instituciones, desde la FDAE se dispone de más de ocho años de experiencia docente en cursos de formación continuada, entre otros, sobre cómo publicar artículos científicos y cómo realizar presentaciones orales en público $[4,5]$. La experiencia se extiende también a la formación de grado [6]. En general, son cursos de dos días de duración, en formato intensivo de mañana y tarde, hasta un total de 15 horas docentes de orientación eminentemente práctica. Se llevan a cabo en grupos reducidos de alumnos, entre 14 y 30 asistentes, y los imparten dos o cuatro profesores, según la modalidad del seminario. La tabla resume los principales cursos y temas organizados hasta la actualidad. Las valoraciones de los cursos realizadas directamente por parte de los asistentes son en general muy positivas [7].

De forma similar a como hicieron GiménezGómez et al [3], desde la FDAE se remitió un cuestionario de opinión por correo electrónico entre los asistentes a los seminarios sobre 'Cómo redactar un artículo científico'. Se pretendía recoger tanto la impresión de los participantes respecto a dicho curso como información sobre los conocimientos aprendidos y propuestas sobre seminarios de formación de cara al futuro [7]. Fue en torno a este último apartado sobre el cual se podrían añadir algunos comentarios, a nuestro parecer interesantes, respecto a las necesidades de formación de los profesionales sanitarios.

Los datos proceden de la información obtenida a partir de los asistentes a 15 ediciones de dichos seminarios, entre 2004 y 2007, que tuvieron lugar en diferentes ciudades españolas y en colaboración con múltiples instituciones. La muestra parte de los 256 asistentes, de los cuales $165(64,5 \%)$ eran mujeres y $91(35,5 \%)$ eran hombres. Se trataba principalmente de médicos, farmacéuticos, enfermeros y biólogos. En total, 76 personas $(29,7 \%)$ respondieron el cuestionario, $51(67,1 \%)$ mujeres y $25(32,9 \%)$ hombres, cuyas necesidades de formación respondieron a la pregunta ¿qué otro tipo de seminarios de formación propondría?’:

- Redacción científica: nivel avanzado/monográficos sobre artículos científicos (ética de la publicación, etc.), cómo redactar otros formatos

\footnotetext{
Fundación Dr. Antonio Esteve.

b Departamento de Ciencias Experimentales y de la Salud. Universitat Pompeu Fabra. Barcelona, España.

Correspondencia Dr. Fèlix Bosch. Fundación Dr. Antonio Esteve. Llobet i Vall-Llosera, 2. E-08032 Barcelona.

Fax +34 934504899 E-mail fbosch@esteve.org
} 
Tabla. Seminarios de formación impartidos por la Fundación Dr. Antonio Esteve desde el año 2001.

\begin{tabular}{|c|c|c|c|}
\hline Contenidos & $\begin{array}{l}\mathrm{N} \cdot{ }^{\circ} \text { de } \\
\text { ediciones }\end{array}$ & Profesores & Objetivos \\
\hline $\begin{array}{l}\text { Cómo redactar } \\
\text { un artículo científico }\end{array}$ & 20 & $\begin{array}{l}\text { E. Fernández } \\
\text { y A.M. García }\end{array}$ & $\begin{array}{l}\text { Dar a conocer las técnicas y habilidades } \\
\text { básicas para escribir y publicar artículos } \\
\text { científicos }\end{array}$ \\
\hline $\begin{array}{l}\text { Cómo realizar presentaciones } \\
\text { orales en biomedicina }\end{array}$ & 12 & $\begin{array}{l}\text { A. Clotet y M. Clotet, } \\
\text { A. Mañas, R. Coma, } \\
\text { M.I. Martín y E. Muñoz }\end{array}$ & $\begin{array}{l}\text { Dar a conocer las técnicas y habilidades } \\
\text { básicas para realizar una presentación oral }\end{array}$ \\
\hline Metodologías docentes: ABP & 9 & $\begin{array}{l}\text { L.A. Branda } \\
\text { y R.M. Torrens }\end{array}$ & $\begin{array}{l}\text { Impartir las bases del ABP y ofrecerlo a } \\
\text { todas aquellas personas interesadas en } \\
\text { este sistema activo de docencia }\end{array}$ \\
\hline $\begin{array}{l}\text { Iniciación al funcionamiento } \\
\text { de los CEIC }\end{array}$ & 7 & $\begin{array}{l}\text { J.E. Baños, I. Galende } \\
\text { y M.I. Lucena }\end{array}$ & $\begin{array}{l}\text { Ofrecer la información mínima necesaria } \\
\text { para aquellos miembros de recién o futura } \\
\text { incorporación a un CEIC }\end{array}$ \\
\hline $\begin{array}{l}\text { Ensayos clínicos en oncología, } \\
\text { para miembros de un CEIC }\end{array}$ & 3 & $\begin{array}{l}\text { M.A. de Cos } \\
\text { y M. Valladares }\end{array}$ & $\begin{array}{l}\text { Revisar los principales aspectos a considerar } \\
\text { durante la evaluación de protocolos de } \\
\text { investigación clínica oncológica }\end{array}$ \\
\hline $\begin{array}{l}\text { Metodologías docentes: } \\
\text { rol del tutor facilitador }\end{array}$ & 1 & $\begin{array}{l}\text { L.A. Branda } \\
\text { y R.M. Torrens }\end{array}$ & $\begin{array}{l}\text { Asistir a los docentes familiarizados con } \\
\text { el ABP a desarrollar habilidades como } \\
\text { tutores facilitadores en las sesiones de ABP }\end{array}$ \\
\hline $\begin{array}{l}\text { s diferentes ediciones de los semir } \\
\text { stituciones (sociedades científicas, } \\
\text { emas; CEIC: comités éticos de inves }\end{array}$ & $\begin{array}{l}\text { s de forma } \\
\text { ersidades, } \mathrm{f} \\
\text { ción clínica. }\end{array}$ & $\begin{array}{l}\text { n tuvieron lugar en disti } \\
\text { ultades, hospitales y cent }\end{array}$ & $\begin{array}{l}\text { dades españolas y con la colaboración de diversas } \\
\text { ención primaria). ABP: aprendizaje basado en pro- }\end{array}$ \\
\hline
\end{tabular}

científicos (capítulos de libro, cartas al director, etc.), análisis de datos y presentación de resultados en artículos, redacción de artículos en publicaciones internacionales.

- Búsqueda de información: Internet y bases de datos biomédicas, revistas médicas de libre acceso, recopilación de documentación y bibliografía.

- Estadística: aplicada a ciencias de la salud (SPSS, SAS, etc.), aplicada a la lectura de un artículo científico.

- Comunicación médico-científica: cómo mejorar los diferentes aspectos de la comunicación científica, elaboración y defensa de un póster científico, realización de ponencias y exposiciones orales, técnicas de comunicación médica (cómo dar una mala noticia, etc.).

- Organización y gestión científica: preparación de un proyecto de investigación, organización de un simposio o minicongreso, gestión de proyectos científicos, coordinación de equipos multidisciplinares, conceptos básicos sobre investigación y desarrollo, obtención de recursos para $\mathrm{I}+\mathrm{D}+\mathrm{I}$ (p. ej., convocatorias públicas), bases para la dirección de un grupo de investigación.

- Ética: relación entre médico e industria farmacéutica, experimentación preclínica y clínica. 
- Investigación de laboratorio: buenas prácticas de investigación, similitudes y desigualdades entre la investigación básica y clínica.

- Miscelánea: epidemiología, presentación de estudios cualitativos, metodología docente en formación y formación de formadores, estudios de uso de medicamentos y de farmacoeconomía, uso racional de medicamentos, cómo aprender a leer un artículo científico.

Aunque se trataba de un perfil de profesionales algo distinto al de Giménez-Gómez et al [3], ambas encuestas presentan ciertas coincidencias en cuanto al tipo de formación solicitada. Por ejemplo, en ambas se reclama formación en torno a la redacción científica, la búsqueda de información, la estadística, la comunicación médico-científica, la epidemiología y los estudios cualitativos. A diferencia del trabajo publicado por Giménez-Gómez et al, a partir de nuestra muestra se sugiere además la necesidad de formación en ética, en organización y gestión científica y en investigación de laboratorio, entre otros. Aunque la necesidad de cursos de inglés científico no fue expresamente especificada por nuestros entrevistados, la demanda de cursos de redacción científica en publicaciones internacionales podría suponer cierta aproximación a dicha necesidad. En nuestro estudio tampoco se demandaba formación sobre programas informáticos tipo Access o PowerPoint, como aparece en el estudio referenciado [3].

Parece evidente que los profesionales sanitarios demandan formación continuada en múltiples temas relacionados con la investigación y la asistencia. Es nuestro deseo que dichas propuestas sirvan como punto de partida para que las instituciones involucradas tomen la iniciativa y organicen cursos bien diseñados y asequibles a fin de cubrir estas necesidades. Dado que parece evidente que existen necesidades, ahora se trataría de ofrecer soluciones.

\section{Bibliografía}

1. Millán Núñez-Cortés J, García-Seoane J, Calvo-Manuel E, Díez-Lobato R, Calvo-Manuel F, Nogales-Espert A. Relaciones de la facultad de medicina con el hospital universitario. La enseñanza de la medicina clínica. Educ Med 2008; 11: 3-6.

2. Clèries X, Martín-Zurro A. La formación actual del médico: fortalezas y debilidades. Educ Med 2009; 12: 7-10.

3. Giménez-Gómez N, Pedrazas-López D, Medina-Rondón E, Dalmau-Juanola D; Grupo de Estudio sobre la Formación en Investigación. Formación en investigación: autopercepción de los profesionales sobre sus necesidades. Med Clin (Barc) 2009; 132: 112-7.

4. Bosch F, Baños JE, Erill S. Medical education beyond patient-physician relationship: an experience from the Esteve Foundation. Educ Med 2004; 7: 47-8.

5. Bosch F, Morales P, Lucena MI, Baños JE. El reto de los cursos de formación para miembros de comités éticos de investigación clínica. Rev And Pat Digest 2005; 28 : 14-20.

6. Rosich L, Baños JE, Bosch F. Del escritorio a la tarima: una experiencia de formación en competencias transversales dentro de la licenciatura en biología. Educ Med 2008; 11: 19-27.

7. Serés E, García AM, Fernández E, Bosch F. Six years of teaching experience in how to write articles. A course of definite interest in science degrees. Meth Find Clin Exp Pharmacol 2009; 31 (Suppl. A): 76. 